\title{
Rana catesbeiana ribonuclease induces cell apoptosis via the caspase-9/-3 signaling pathway in human glioblastoma DBTRG, GBM8901 and GBM8401 cell lines
}

\author{
JEN-NI CHEN ${ }^{1,2^{*}}$, GIOU-TENG YIANG ${ }^{3,4 *}$, YI-FAN LIN ${ }^{5}$, PEI-LUN CHOU ${ }^{6,7}$, TSAI-KUN WU $^{8,9}$, \\ WEI-JUNG CHANG ${ }^{10}$, CHINSHUH CHEN $^{1}$ and YUNG-LUEN YU ${ }^{8,10,11}$
}

\begin{abstract}
${ }^{1}$ Department of Food Science and Biotechnology, National Chung Hsing University, Taichung $402 ;{ }^{2}$ Department of Nutrition, Master Program of Biomedical Nutrition, Hungkuang University, Taichung 433; ${ }^{3}$ Department of Emergency Medicine, Taipei Tzu Chi Hospital, Buddhist Tzu Chi Medical Foundation, New Taipei 231; ${ }^{4}$ Department of Emergency Medicine, School of Medicine, Tzu Chi University, Hualien 970; ${ }^{5}$ Department of Medical Research, Taipei Veterans General Hospital, Taipei 112; ${ }^{6}$ Division of Allergy-Immunology-Rheumatology, Department of Internal Medicine,

Saint Mary's Hospital Luodong, Luodong, Yilan 265; ${ }^{7}$ Department of Internal Medicine, School of Medicine, College of Medicine, Taipei Medical University, Taipei 110; ${ }^{8} \mathrm{The}$ PhD Program for Cancer Biology and Drug Discovery, China Medical University, Taichung 404; ${ }^{9}$ Division of Renal Medicine, Tungs' Taichung MetroHarbor Hospital, Taichung 435;

${ }^{10}$ Graduate Institute of Cancer Biology, Center for Molecular Medicine, China Medical University, Taichung 404;

${ }^{11}$ Department of Biotechnology, Asia University, Taichung 413, Taiwan, R.O.C.
\end{abstract}

Received May 27, 2014; Accepted February 17, 2015

DOI: $10.3892 / \mathrm{ol} .2015 .3117$

\begin{abstract}
Human glioblastoma multiforme is one of the most aggressive malignant brain tumor types, and the mean survival time of patients with a brain tumor is $<2$ years when traditional therapies are administered. Thus, numerous studies have focused on the development of novel treatments for brain tumors. Frog ribonucleases, such as Onconase and Rana catesbeiana ribonuclease (RC-RNase), exert antitumor effects on various tumor cells, including cervical cancer, breast cancer, hepatoma, leukemia, pancreatic cancer and prostate cancer cells. In addition, frog Onconase has been applied as a treatment in clinical trials. However, the antitumor effects of frog ribonucleases on brain tumors are unclear. Previous studies have indicated that RC-RNase demonstrates a decreased cytotoxic effect in normal cells compared with Onconase. Therefore, the present study investigated the ability of RC-RNase to exert antitumor activities on human glioblastoma.
\end{abstract}

Correspondence to: Professor Yung-Luen Yu, Graduate Institute of Cancer Biology, Center for Molecular Medicine China Medical University, 91 Hsueh-Shih Road, Taichung 404, Taiwan, R.O.C.

E-mail: ylyu@mail.cmu.edu.tw

Dr Chinshuh Chen, Department of Food Science and Biotechnology, National Chung Hsing University, 250 Kuokuang Road, Taichung 402, Taiwan

E-mail: chinshuh@dragon.nchu.edu.tw

*Contributed equally

Key words: frog ribonulease, brain tumor, apoptosis, caspase
It was found that RC-RNase inhibits the growth of the human glioblastoma DBTRG, GBM8901 and GBM8401 cells. In addition, the present study revealed that RC-RNase induces caspase-9/-3 activity and triggers the apoptotic cell death pathway in human glioblastoma cells. Notably, it was also demonstrated that RC-RNase effectively inhibits the growth of human glioblastoma tumors in a nude mouse model. Overall, the present study indicates that RC-RNase may be a potential agent for the treatment of human glioblastoma.

\section{Introduction}

Human glioblastoma multiforme (GBM) is one the most malignant types of brain tumor. In general, the traditional tumor therapies, consisting of surgery, chemotherapy, radiotherapy and a combination of these methods, are applied for the clinical treatment of GBM (1-3). However, the mean survival time of patients with GBM is $<2$ two years $(2,3)$. Therefore, potential therapies for the treatment of patients with brain tumors are required and have previously been investigated (4-6).

Rana catesbeiana ribonuclease (RC-RNase) and Onconase are cytotoxic ribonucleases that are purified from frog oocyte $(7,8)$. Onconase, which is derived from Rana pipiens, and RC-RNase, which is derived from Rana catesbeiana, each belong to the RNase A superfamily $(9,10)$. RC-RNase and Onconase have been revealed to demonstrate antitumor activity (10-13). Previous studies have reported that RC-RNase is able to exert cytotoxic effects in various tumor cells, including cervical cancer, leukemia, hepatoma and breast cancer cells $(11,14-16)$. In addition, numerous studies have indicated that RC-RNase is able to trigger distinct cell death mechanisms in various tumor cell types $(10,11,14,17)$. By contrast, previous 
studies have also revealed that Onconase can exert antitumor activities in numerous tumor cells, including cervical, breast, colon, pancreatic and prostate cancer cells $(12,18-22)$.

As previous studies have demonstrated, RC-RNase and Onconase exert antitumor activities in various human tumors $(17,23,24)$. In particular, Onconase has been used as an antitumor agent in clinical trials $(7,19,25)$. Additionally, a previous study reported that Onconase exerts a cytotoxic effect on the human glioma SHG-44 cell line (26). This result indicated that frog ribonuclease may be a potential antitumor agent for the therapy of brain tumors arising from glial cells. By contrast, it is notable that Onconase and RC-RNase each exert antitumor effects in numerous tumor cell lines, while RC-RNase exerts a decreased cytotoxic effect in normal cells compared with Onconase (10). Therefore, the present study investigates the ability of RC-RNase to demonstrate antitumor effects in glioblastoma cells.

\section{Materials and methods}

Reagents. RC-RNase was provided by Dr Jaang-Jiun Wang (Division of Pediatric Infectious Diseases, Emory University School of Medicine, Atlanta, GA, USA). The MTT assay kit was obtained from Bio Basic, Inc. (Markham, ON, Canada). The Hoechst 33342 stain was purchased from Sigma-Aldrich (St. Louis, MO, USA). The caspase-3 substrate acetyl-Asp-Glu-Val-Asp p-nitroanilide (Ac-DEVD-pNA), caspase-8 substrate Ac-Ile-Glu-Thr-Asp pNA (Ac-IETD-pNA), and caspase-9 substrate Ac-Leu-Glu-His-Asp pNA (Ac-LEHD-pNA) were purchased from Anaspec, Inc. (Fremont, CA, USA). Fetal bovine serum, Dulbecco's modified Eagle's medium (DMEM), non-essential amino acids, L-glutamine and penicillin-streptomycin were purchased from Gibco Life Technologies (Carlsbad, CA, USA).

Cell lines and cell culture. The human glioblastoma DBTRG, GBM8901 and GBM8401 and rat glioblastoma RG2 cell lines were purchased from Bioresources Collection and Research Center (Food Industry Research and Development Institute, Hsinchu, Taiwan). The DBTRG, GBM8901 and GBM8401 cell lines were cultured in RPMI medium containing $10 \%$ fetal bovine serum, $2 \mathrm{mM}$ L-glutamine, 100 international units (IU)/ml penicillin-streptomycin and $0.1 \mathrm{mM}$ non-essential amino acids. The RG2 cell line was cultured in DMEM containing 10\% fetal bovine serum, $2 \mathrm{mM}$ L-glutamine, $100 \mathrm{IU} / \mathrm{ml}$ penicillin-streptomycin and $0.1 \mathrm{mM}$ non-essential amino acids. The cells were maintained at $37^{\circ} \mathrm{C}$ in a humidified $5 \% \mathrm{CO}_{2}$ atmosphere.

Inhibition rate assay. Cell viability was determined using an MTT assay, according to the manufacturer's instructions. In brief, 4,000 cells per well were cultured in each well of a 96-well culture dish. Every $24 \mathrm{~h}$, the control group (cell without RC-RNase treatment) and experimental groups (cells with 2, 20 and $50 \mu \mathrm{g} / \mathrm{ml}$ RC-RNase treatment) were treated using the MTT assay kit and the cell viability was observed for $96 \mathrm{~h}$. After $3 \mathrm{~h}$ of reaction, the absorbance at $570 \mathrm{~nm}$ for each well was measured under a multi-well ELISA reader (Molecular Devices LLC, Sunnyvale, CA, USA). The inhibition rate was calculated using the following formula:

$$
\text { Inhibition rate }(\%)=100-\frac{A_{570 \text { experimental }}}{A_{570 \text { control }}} \times 100
$$

Observation of nuclear condensation. Nuclear condensation is an apoptotic characteristic that may be determined using a nuclear staining method $(11,27)$. Briefly, subsequent to the cells being treated with $10 \mu \mathrm{g} / \mathrm{ml}$ Hoechst 33342 for $10 \mathrm{~min}$, nuclear condensation was observed under a fluorescence microscope with an excitation wavelength of $352 \mathrm{~nm}$ and an emission wavelength of $450 \mathrm{~nm}$.

Sub- $G_{I}$ phase analysis. The accumulation of cells in the sub- $\mathrm{G}_{1}$ phase is an apoptotic characteristic and may be determined using a fluorescence-activated cell sorting (FACS) method, as previously described (28-30). Briefly, control (DBTRG cells without RC-RNase treatment) and experimental cells (DBTRG cells with $20 \mathrm{ug} / \mathrm{ml}$ RC-RNase treatment) were fixed with $70 \%$ alcohol at $4^{\circ} \mathrm{C}$ for $1 \mathrm{~h}$. The fixed cells were washed with phosphate-buffered saline (PBS) and treated with $1 \mathrm{ml}$ propidium iodide (PI) solution, containing $50 \mu \mathrm{g} / \mathrm{ml} \mathrm{PI}$, $100 \mu \mathrm{g} / \mathrm{ml}$ RNase A and $0.1 \%$ Triton X-100, at $37^{\circ} \mathrm{C}$ for $1 \mathrm{~h}$. Following the PBS wash, these cells were analyzed by flow cytometry, which was performed using the CyFlow SL flow cytometer and Flomax software from Sysmex Partec GmbH (Görlitz, Görlitz, Germany). The data were calculated using WinMDI software, version 2.8 (The Scripps Research Institute, La Jolla, CA, USA).

Caspase activity assay. The caspase activity assay was performed as previously described $(9,11,27)$. In brief, the cells were treated with a lysis buffer, consisting of $50 \mathrm{mM}$ Tris-HCl, $120 \mathrm{mM} \mathrm{NaCl}, 1 \mathrm{mM}$ EDTA and $1 \%$ nonyl phenoxypolyethoxylethanol-40 ( $\mathrm{pH} 7.5)$, supplemented with protease inhibitors. Cell pellets were collected subsequent to centrifugation at $15,000 \mathrm{x} \mathrm{g}$ for $30 \mathrm{~min}$ at $4^{\circ} \mathrm{C}$. In total, $40 \mu \mathrm{l}$ of cell lysate, containing $80 \mu \mathrm{g}$ total protein, was added to the reaction solution containing $158 \mu \mathrm{l}$ reaction buffer consisting of $20 \%$ glycerol, $0.5 \mathrm{mM}$ EDTA, $5 \mathrm{mM}$ dithiothreitol and $100 \mathrm{mM}$ HEPES (pH 7.5), and $2 \mu 1$ fluorogenic caspase substrate (Ac-LEHD-pNA, Ac-DEVD-pNA or Ac-IETD-pNA). The combined cell lysate and reaction mixture was then incubated at $37^{\circ} \mathrm{C}$. Subsequent to a 6-h reaction period, the fluorogenic substrate was detected at $405 \mathrm{~nm}$ in an ultra-microplate reader (Bio-Tek Instruments, Inc., Winooski, VT, USA). The fold increase of caspase activity was calculated using the following formula:

$$
\text { Fold increase }=\frac{\mathrm{A}_{405} \text { experimental }}{\mathrm{A}_{405 \text { control }}} \times 100
$$

Mouse weight observation. In total, 16 four-week-old female nude mice were obtained from the National Laboratory Animal Center (Taipei, Taiwan). All mice were divided into two groups, with eight mice per group. One group was treated with $100 \mu \mathrm{l}$ RC-RNase at a concentration of $50 \mu \mathrm{g} / \mathrm{ml}$, and the other group was treated with $100 \mu 1$ PBS. Every three days, the weight of the mice was measured using an electronic weighing scale. This procedure was approved by the Animal Committee of the Tzu Chi University (Hualien, Taiwan). 
Animal studies. In total, 16 four-week-old female nude mice were obtained from the National Laboratory Animal Center. All mice were divided into two groups, with eight mice per group, and were injected with $10^{7}$ DBTRG cells in the lower abdominal region. After three weeks, one group of mice was injected with $100 \mu \mathrm{l}$ RC-RNase at a concentration of $50 \mu \mathrm{g} / \mathrm{ml}$ on the opposite side of the lower abdominal region to the tumor. The second group was injected with $100 \mu \mathrm{l}$ PBS on the opposite side of the lower abdominal region to the tumor. Day zero was classified as the day that treatment with RC-RNase and PBS commenced. Every three days the tumor size was calculated according to the following formula, where A was the greater and B was the smaller of the two measured dimensions of the tumor: Tumor size $=\left(\mathrm{A} \mathrm{x} \mathrm{B}^{2}\right) / 2$. This procedure was approved by the animal committee of the Tzu-Chi University (Hualien, Taiwan).

Statistical analysis. Student's $t$-test was utilized to analyze the present data, which was presented as the mean \pm standard error. $\mathrm{P}<0.1$ was considered to indicate a statistically significant difference.

\section{Results}

$R C$-RNase triggers antitumor effects in human glioblastoma cells. Previous studies have demonstrated that RC-RNase possesses antitumor properties in various tumors $(10,11,16,17)$. Notably, it has been revealed that RC-RNase is not cytotoxic to normal cells $(10,15)$. However, the ability of RC-RNase to inhibit glioblastoma cell growth is not clear. In the present study, the human glioblastoma DBTRG, GBN8901 and GBM8401 and the rat glioblastoma RG2 cell lines were treated with $20 \mu \mathrm{g} / \mathrm{ml}$ RC-RNase (Fig. 1A). The data revealed that RC-RNase inhibits the growth of human glioblastoma cells. However, the inhibition rate demonstrated by RC-RNase-treated RG2 cells was decreased compared with the rate exhibited by the other cell lines. These results indicated that RC-RNase triggers cytotoxic effects more strongly in human glioblastoma cells compared with rat glioblastoma cells. In addition, the human glioblastoma cells were also treated with various concentrations of RC-RNase, consisting of 2, 20 and $50 \mu \mathrm{g} / \mathrm{ml} \mathrm{RC-RNase.}$ As Fig. 1B shows, RC-RNase exerts a dose-dependent antitumor effect on DBTRG cells. Similar results were found in RC-RNase-treated GBM8901 and GBM8401 cells (data not shown).

RC-RNase induces apoptosis and activation of caspases-9 and -3 in DBTRG cells. The present study further determined whether RC-RNase is able to induce apoptotic cell death in human glioblastoma cells. Nuclear condensation was an apoptotic characteristic and could be determined using the nuclear staining method $(11,27)$. The DBTRG cells were treated with $50 \mu \mathrm{g} / \mathrm{ml}$ RC-RNase for $72 \mathrm{~h}$, and the cell morphology was observed under a a florescent microscope (excitation, 352; emission, 450 nm; Olympus BX61; Olympus Corporation, Tokyo, Japan) (Fig. 2). By contrast to the control cells (Fig. 2C), nuclear condensation was found in RC-RNase-treated cells (Fig. 2D). Therefore, RC-RNase may induce apoptotic cell death in DBTRG cells. Additionally, the apoptotic characteristic of cells accumulating in the sub- $\mathrm{G}_{1}$ phase was identified following $72 \mathrm{~h}$ of treatment in the RC-RNase-treated DBTRG cells, which
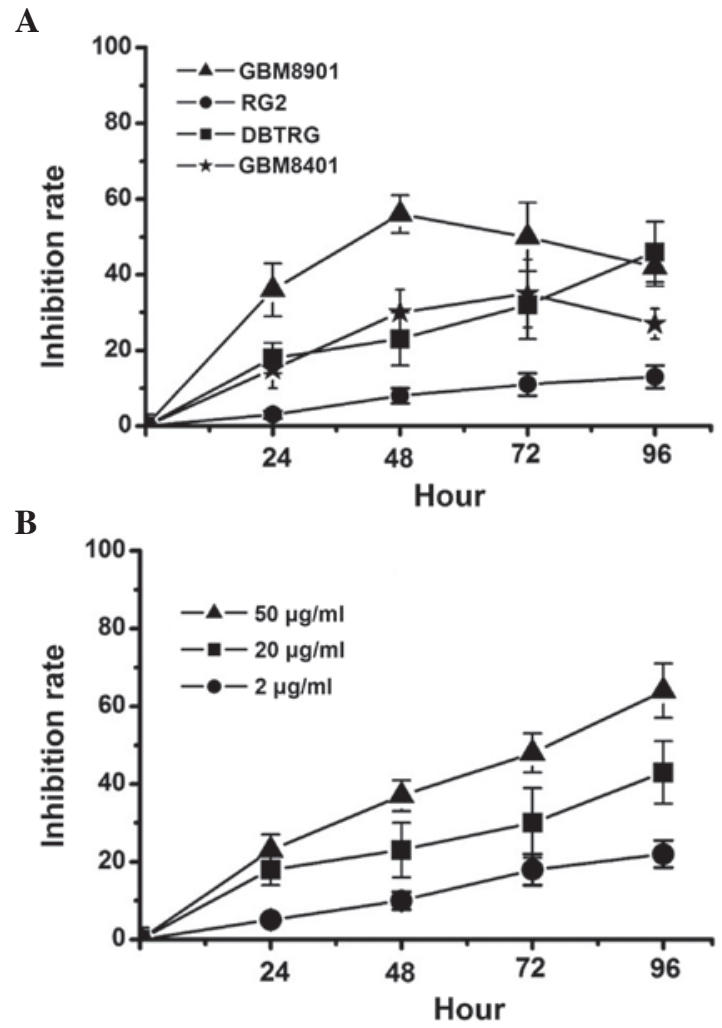

Figure 1. Inhibition rate of Rana catesbeiana ribonuclease (RC-RNase) in glioblastoma cell lines. (A) Human glioblastoma GBM8901, GBM8401 and DBTRG and rat glioblastoma RG2 cells were treated with $20 \mu \mathrm{g} / \mathrm{ml}$ RC-RNase. (B) DBTRG cells were treated with 2,20 and 50 $\mu \mathrm{g} / \mathrm{ml}$ RC-RNase. The inhibiton of cells was measured every $24 \mathrm{~h}$. The data were obatined from four triplicate groups and expressed as the mean \pm standard deviation.

was determined using a FACS analysis method as previously described (28-30). As Fig. 3 shows, $3 \%$ of control cells were in the sub- $\mathrm{G}_{1}$ phase, whereas $\sim 30 \%$ of RC-treated DBTRG cells were in the sub- $\mathrm{G}_{1}$ phase. Overall, these data indicate that $\mathrm{RC}-\mathrm{RNase}$ induces apoptosis in human glioblastoma cells.

RC-RNase activates caspases-9 and -3 in DBTRG cells. The apoptotic cell death pathway can be divided into the caspase-dependent and caspase-independent pathways $(27,31,32)$. Therefore, the present study further determined the induction of caspase activation in RC-RNase-treated cells. The activity of caspases-3, -8 and -9 was determined using a substrate cleavage assay, as previously described $(9,10,16)$. The present data revealed that the activity of caspases-3 and -9 was increased in RC-RNase-treated DBTRG cells at $72 \mathrm{~h}$ compared with the PBS-treated cells (Fig. 4A and B). However, the activity of caspase- 8 was not evidently increased in RC-RNase-treated DBTRG cells compared with the PBS-treated cells (Fig. 4C). Previous studies have indicated that the caspase-dependent apoptotic cell death pathway is markedly induced through the mitochondrial pathway, caspase-9/-3 cascade, death receptor pathway or caspase-8/-3 cascade $(10,16)$. Therefore, the present results suggested that RC-RNase induces apoptosis in the DBTRG cells through the mitochondrial pathway.

$R C$-RNase effectively inhibits the growth of glioblastoma cells in a nude mouse model. The present results demonstrated that RC-RNase exerts antitumor effects in glioblastoma cells in a 

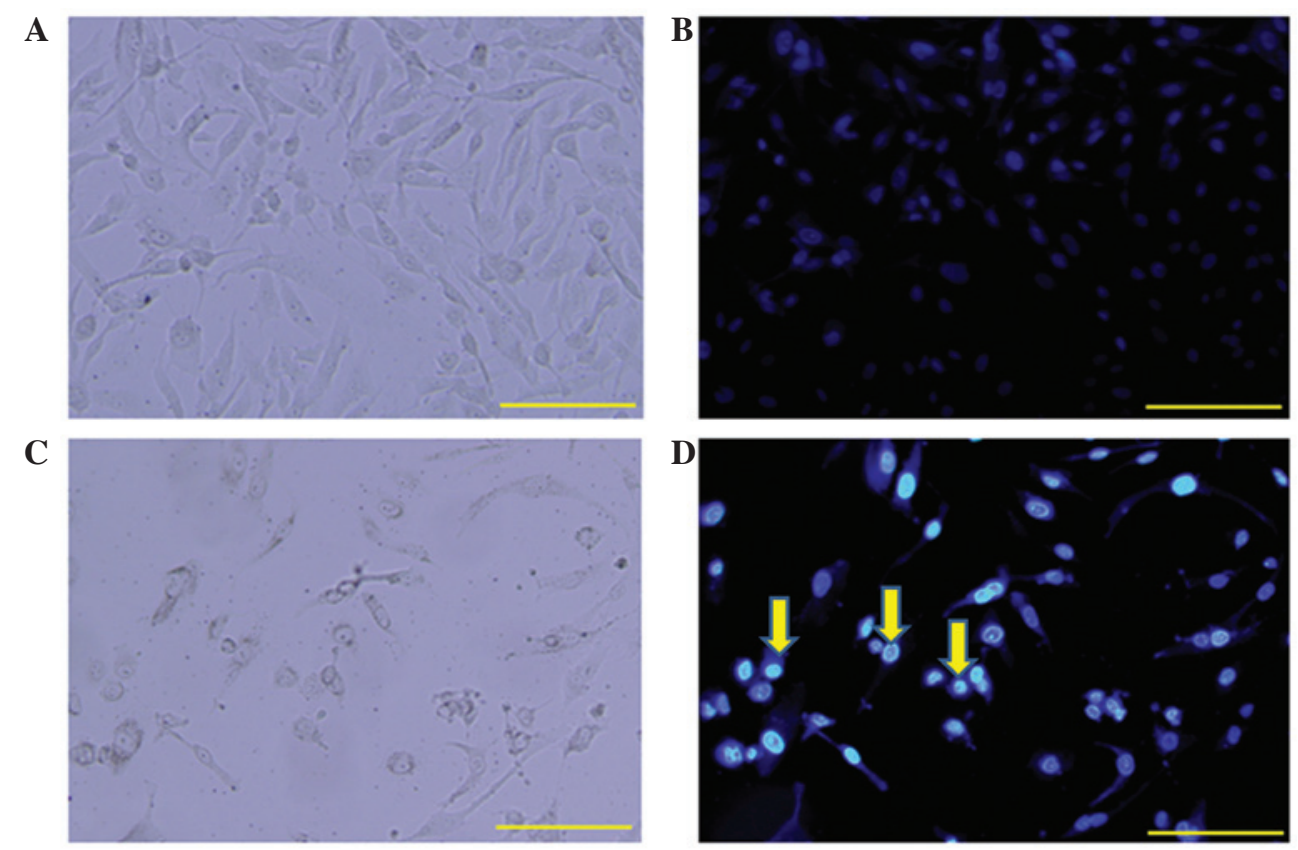

Figure 2. Cell morphology of (A and C) control phosphate-buffered saline-treated cells and (B and D) cells treated with $50 \mu \mathrm{M}$ Rana catesbeiana ribonuclease (RC-RNase) at $72 \mathrm{~h}$. (A and B) The cells were observed under a phase-contrast microscope. (C and D) The cells were observed under a florescent microscope. Nuclear condensation (yellow arrow) was identified in RC-RNase-treated cells. Scale bar, $100 \mu \mathrm{m}$.

A

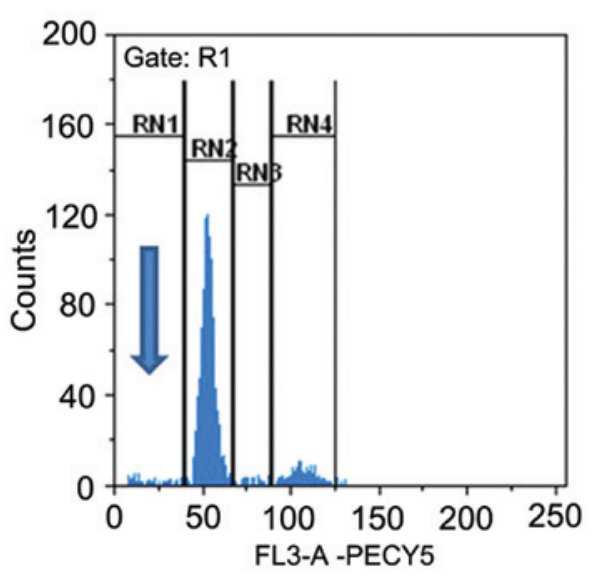

B

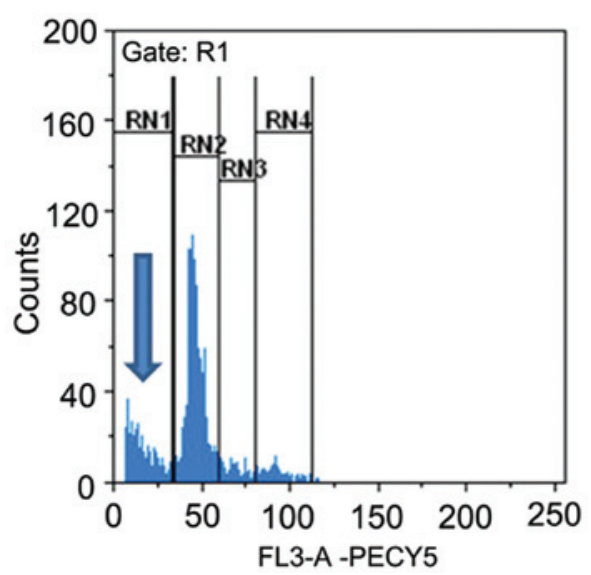

Figure 3. Sub-G $\mathrm{G}_{1}$ phase analysis in (A) control phosphate-buffered saline-treated cells and (B) Rana catesbeiana ribonuclease-treated cells at $72 \mathrm{~h}$. The cell cycle was analyzed by flow cytometry. Sub-G $\mathrm{G}_{1}$ phase was marked at the RN1 area (blue arrows). Approximately $30 \%$ of RC-RNasetreated cells can been observed in Sub G1 phase. cell model. In addition, the present study further investigated the inhibitory ability of RC-RNase on the growth of glioblastoma cells in an animal model. In order to assess the toxic effects of RC-RNase on nude mice, the weight of the mice was observed in RC-RNase-treated and PBS-treated mice (Fig. 5). The present data revealed that there was no evident difference between the weight of the RC-RNase-treated and PBS-treated mice. Subsequently, the growth of glioblastoma cells was measured in RC-RNase-treated and PBS-treated mice. The DBTRG cells were first injected subcutaneously into the lower abdominal region of the mice. After three weeks, the tumor size had grown to $\sim 5 \mathrm{~mm}^{3}$, and the mice were administered with either RC-RNase or PBS through a subcutaneous injection to the opposite side of the abdominal region to the tumor. Prior to day 15 of treatment, the tumor size was not significantly different between the RC-RNase-treated and PBS-treated mice (Fig. 6). However, the tumor size was smaller in RC-RNase-treated mice compared with the PBS-treated mice after 15 days of RC-RNase or PBS administration (Fig. 6). The present study suggested that RC-RNase exerted antitumor effects on human glioblastoma cells in an animal model. In summary, the present study demonstrated that RC-RNase possess antitumor properties when administered to human glioblastoma cells in cell and animal models. In addition, RC-RNase is able activate the activity of caspases- -9 and -3 and induce apoptosis on human glioblastoma cells.

\section{Discussion}

In order to determine whether RC-RNase exerted antitumor effects on glioblastoma cells, the rat RG2 and human DBTRG, GBM8901 and GBM8401 glioblastoma cell lines were treated with RC-RNase in the present study (Fig. 1A). The present data revealed that RC-RNase exerted a stronger cytotoxic 
A

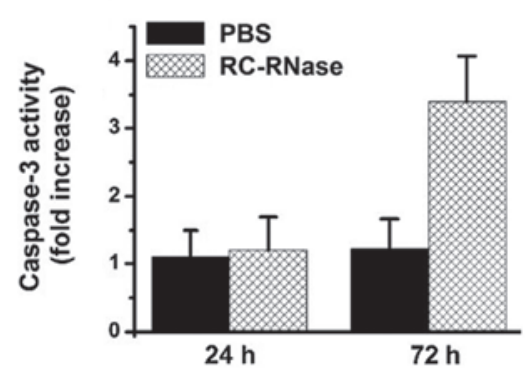

B

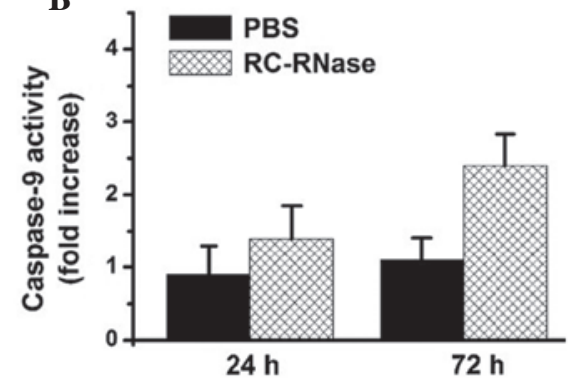

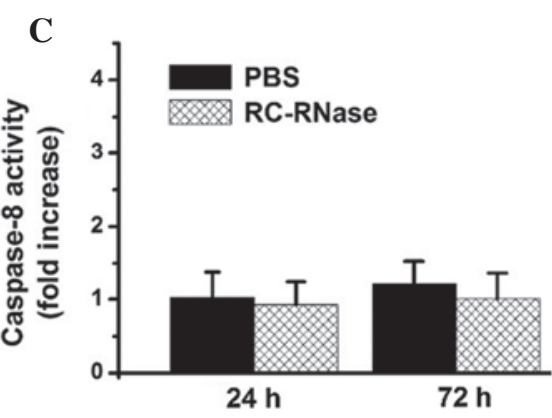

Figure 4. Activity of (A) caspase-3, (B) caspase-9 and (C) caspase-8 measured in PBS-treated and $50 \mu \mathrm{g} / \mathrm{ml}$ RC-RNase-treated DBTRG cells at the indicated time points ( 24 and $72 \mathrm{~h}$ ). The activity of caspase-3 and caspase- 9 was increased in RC-RNase-treated cells compared with PBS-treated cells. The data was obtained from three independent experiments and expressed as the mean \pm standard deviation. The data suggested that casase- 3 and caspase- 9 were significantly activated in RC-RNase-treated cells at $72 \mathrm{~h}$. PBS, phosphate-buffered saline; RC-RNase, Rana catesbeiana ribonuclease.

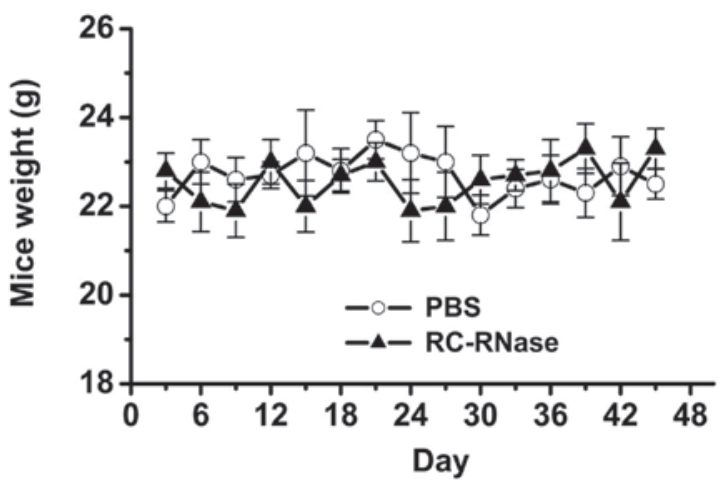

Figure 5. Mouse weight. Eight mice were treated with $50 \mu \mathrm{g} / \mathrm{ml}$ RC-RNase and eight mice were treated with PBS. (The volumns of RC-RNase and PBS are all $100 \mu \mathrm{l}$ ) The weight of the mice was measured every three days. There was no significant difference between the weight of the RC-RNase-treated and PBS-treated mice. PBS, phosphate-buffered saline; RC-RNase, Rana catesbeiana ribonuclease.

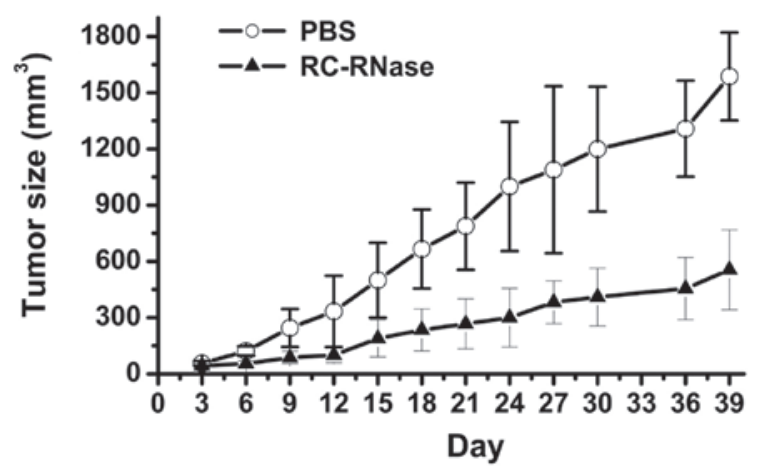

Figure 6. Tumor size. Subsequent to the DBTRG cells being injected subcutaneously into the lower abdominal region of the mice, the mice did not undergo treatment for three weeks. The following day, indicated as day zero, $50 \mu \mathrm{g} / \mathrm{ml}$ RC-RNase and PBS were injected subcutaneously in the opposite side of the abdominal region to the tumor (The volumns of RC-RNase and PBS were $100 \mu$ l). Tumor size was measured every three days. Eight mice were examined for each group. The data indicate that the tumor size was significantly different after 18 days of RC-RNase treatment. PBS, phosphate-buffered saline; RC-RNase, Rana catesbeiana ribonuclease.

effect on human glioblastoma cell lines, but did not exert an evident cytotoxic effect on the rat glioblastoma cell line. This result indicates that RC-RNase-induced cytotoxic effects may be associated with the animal species. In addition, the data obtained on the inhibition rate of RC-RNase on the growth of the three human glioblastoma cell lines revealed that RC-RNase inhibited the GBM 8401 cells more strongly than the GBM8901 and DBTRG cells. By contrast, as shown in Fig. 1A, the inhibition rate continually increased with dose in RC-RNase-treated DBTRG cells. However, the inhibition rate did not continually increase with dose in the RC-RNase-treated GBM8401 and GBM8901 cells. These results suggest that a number of the GBM8401 and GBM8901 cells may be resistant to RC-RNase. However, a previous study has revealed that Onconase can induce cytotoxic effects in the human glioma SHG-44 cell line (26), and the present study demonstrated that RC-RNase can exert cytotoxic effects on the human glioblastoma DBTRG, GBM8901 and GBM8401 cell lines. Overall, these studies suggest that frog ribonucleases exert potential antitumor effects on glioblastoma cells.

Previous studies have reported that apoptotic characteristics and autophagocytosis can be identified in RC-RNase-treated breast tumor MCF-7 cells (11), while no evident apoptotic and necrotic characteristics are observed in RC-RNase-treated hepatoma SK-Hep1, HepG2 and J5 cells (14). As with RC-RNase-treated MCF-7 cells, apoptotic characteristics were also identified in RC-RNase-treated glioblastoma cells in the present study. However, autophagocytosis was not observed in RC-RNase-treated glioblastoma cells. By contrast, numerous studies have demonstrated that caspase activity is induced in RC-RNase-treated cells $(9,10,16,17)$. RC-RNase can activate different caspases signals to induce cell apoptosis. Certain studies have revealed that caspases- $9,-8$ and -3 can be activated in RC-RNase-treated HL-60 cells $(9,10,16)$. In addition, previous studies have reported that casapses-7, -2 and -6 are activated while caspase- 9 and -8 are not activated in RC-RNase-treated MCF-7 cells $(10,17)$. However, another study indicated that no evident caspase activity is found in RC-RNase-treated SK-Hep 1 cells (10). Currently, the present results demonstrated that the activity of caspases- 9 and -3 is activated in RC-RNase-treated glioblastoma cells, while the activity of caspse- 8 is not activated. Overall, the findings of the aforementioned and present studies suggest that RC-RNase exerts antitumor effects on various tumor cell lines through different cell death signals.

Although numerous studies have demonstrated that frog ribonucleases, including Onconase and RC-RNase, exert antitumor effects on various tumor cell types, only one study 
has indicated that Onconase may inhibit cell growth in the glioma SHG-44 cell line (26). In the present study, the results demonstrated that RC-RNase exerts antitumor activity on the human glioblastoma DBTRG, GBM8901 and GBM8401 cell lines. Furthermore, at present no study has demonstrated that frog ribonuclease inhibits glioblastoma growth in animal models. The current study, to the best of our knowledge, is the first to demonstrate that RC-RNase can effectively inhibit human glioblastoma growth in a nude mouse model. The present results indicated that frog ribonucleases may potentially be used as antitumor agents for glioblastoma treatment. However, the ability of frog ribonucleases to pass through the blood-brain barrier (BBB) remains to be investigated in the future. If frog ribonucleases are able to pass through the BBB, these agents may be used directly for glioblastoma therapy. However, if frog ribonucleases are unable to pass through the $\mathrm{BBB}$, the agents may be administered with surgical treatment for glioblastoma therapy.

In summary, the present study was, to the best of our knowledge, the first to demonstrate that RC-RNase is able to induce the activity of caspase- 9 and -3 in order to induce cell apoptosis, and exert antitumor effects on glioblastoma cells and lesions in cell and animal models.

\section{Acknowledgements}

This research is supported in part by the Ministry of Science and Technology (grant no. MOST 103-2320-B-039-052-MY3); National Health Research Institutes (grant no. NHRI-EX102-10245BI); and Taipei Tzu Chi Hospital (grant nos. TCRD-TPE-102-26; TCRD-TPE-103-48).

\section{References}

1. Afra D, Osztie E, Sipos L and Vitanovics D: Preoperative history and postoperative survival of supratentorial low-grade astrocytomas. Br J Neurosurg 13: 299-305, 1999.

2. Daneyemez M, Gezen F, Canakçi Z and Kahraman S: Radical surgery and reoperation in supratentorial malignant glial tumors. Minim Invasive Neurosurg 41: 209-213, 1998.

3. Yiang GT, Chou RH, Chang WJ, et al: Long-term expression of rAAV2-hIL15 enhances immunoglobulin production and lymphokine-activated killer cell-mediated human glioblastoma cell death. Mol Clin Oncol 1: 321-325, 2013.

4. Fève M, Saliou JM, Zeniou M, et al: Comparative expression study of the endo-G protein coupled receptor (GPCR) repertoire in human glioblastoma cancer stem-like cells, U87-MG cells and non malignant cells of neural origin unveils new potential therapeutic targets. PLoS ONE 9: e91519, 2014.

5. Hegde M, Bielamowicz KJ and Ahmed N: Novel approaches and mechanisms of immunotherapy for glioblastoma. Discov Med 17: 145-154, 2014.

6. Qin Y, Fu M, Takahashi M, et al: Epithelial membrane protein-2 (EMP2) activates Src protein and is a novel therapeutic target for glioblastoma. J Biol Chem 289: 13974-13985, 2014.

7. Ardelt W, Shogen K and Darzynkiewicz Z: Onconase and amphinase, the antitumor ribonucleases from Rana pipiens oocytes. Curr Pharm Biotechnol 9: 215-225, 2008.

8. Ogawa Y, Sugawara S, Tatsuta T, et al: Sialyl-glycoconjugates in cholesterol-rich microdomains of P388 cells are the triggers for apoptosis induced by Rana catesbeiana oocyte ribonuclease. Glycoconj J 31: 171-184, 2014.

9. Yiang GT, Yu YL, Hu SC, et al: PKC and MEK pathways inhibit caspase-9/-3-mediated cytotoxicity in differentiated cells. FEBS Lett 582: 881-885, 2008.

10. Tang CH, Hu CC, Wei CW and Wang JJ: Synergism of Rana catesbeiana ribonuclease and IFN-gamma triggers distinct death machineries in different human cancer cells. FEBS Lett 579: 265-270, 2005 .
11. Yiang GT, Yu YL, Chou PL, et al: The cytotoxic protein can induce autophagocytosis in addition to apoptosis in MCF-7 human breast cancer cells. In Vivo 26: 403-409, 2012.

12. Chang CH, Gupta P, Michel R, et al: Ranpirnase (frog RNase) targeted with a humanized, internalizing, anti-Trop-2 antibody has potent cytotoxicity against diverse epithelial cancer cells. Mol Cancer Ther 9: 2276-2286, 2010.

13. Zhao HL, Xue C, Du JL, et al: Sustained and cancer cell targeted cytosolic delivery of Onconase results in potent antitumor effects. J Control Release 159: 346-352, 2012.

14. Hu CC, Lee YH, Tang CH, et al: Synergistic cytotoxicity of Rana catesbeiana ribonuclease and IFN-gamma on hepatoma cells. Biochem Biophys Res Commun 280: 1229-1236, 2001.

15. Liao YD, Huang HC, Leu YJ, et al: Purification and cloning of cytotoxic ribonucleases from Rana catesbeiana (bullfrog). Nucleic Acids Res 28: 4097-4104, 2000.

16. Wei CW, Hu CC, Tang CH, et al: Induction of differentiation rescues HL-60 cells from Rana catesbeiana ribonuclease-induced cell death. FEBS Lett 531: 421-426, 2002.

17. Hu CC, Tang CH and Wang JJ: Caspase activation in response to cytotoxic Rana catesbeiana ribonuclease in MCF-7 cells. FEBS Lett 503: 65-68, 2001.

18. Zwolińska M and Smolewski P: Onconase: A ribonuclease with antitumor activity. Postepy Hig Med Dosw (Online) 64: 58-66, 2010 (In Polish).

19. Rybak SM, Arndt MA, Schirrmann T, Dübel S and Krauss J: Ribonucleases and immunoRNases as anticancer drugs. Curr Pharm Des 15: 2665-2675, 2009.

20. Castro J, Ribó M, Puig T, et al: A cytotoxic ribonuclease reduces the expression level of P-glycoprotein in multidrug-resistant cell lines. Invest New Drugs 30: 880-888, 2012.

21. Halicka HD, Murakami T, Papageorgio CN, et al: Induction of differentiation of leukaemic (HL-60) or prostate cancer (LNCaP, JCA-1) cells potentiates apoptosis triggered by onconase. Cell Prolif 33: 407-417, 2000.

22. Ita M, Halicka HD, Tanaka T, et al: Remarkable enhancement of cytotoxicity of onconase and cepharanthine when used in combination on various tumor cell lines. Cancer Biol Ther 7: 1104-1108, 2008

23. Ardelt B, Juan G, Burfeind P, et al: Onconase, an anti-tumor ribonuclease suppresses intracellular oxidative stress. Int J Oncol 31: 663-669, 2007.

24. Fang EF and Ng TB: Ribonucleases of different origins with a wide spectrum of medicinal applications. Biochim Biophys Acta 1815: 65-74, 2011.

25. Castro J, Ribó M, Navarro S, et al: A human ribonuclease induces apoptosis associated with p21WAF1/CIP1 induction and JNK inactivation. BMC Cancer 11: 9, 2011.

26. Wang XM and Guo ZY: Recombinant expression, different downstream processing of the disulfide-rich anti-tumor peptide Ranpirnase and its effect on the growth of human glioma cell line SHG-44. Biomed Rep 1: 747-750, 2013.

27. Yiang GT, Chen YH, Chou PL, et al: The NS3 protease and helicase domains of Japanese encephalitis virus trigger cell death via caspase dependent and independent pathways. Mol Med Rep 7: 826-830, 2013

28. Shin JS, Maeng HG, Hong SW, et al: Iris Nertschinskia ethanol extract differentially induces cy totoxicity in human breast cancer cells depending on AKT1/2 activity. Asian Pac J Cancer Prev 13: 6511-6516, 2012.

29. Nho KJ, Chun JM and Kim HK: Agrimonia pilosa ethanol extract induces apoptotic cell death in HepG2 cells. J Ethnopharmacol 138: 358-363, 2011.

30. Yu YL, Yu SL, Su KJ, et al: Extended O6-methylguanine methyltransferase promoter hypermethylation following n-butylidenephthalide combined with 1,3-bis(2-chloroethyl)-1-nitrosourea (BCNU) on inhibition of human hepatocellular carcinoma cell growth. J Agric Food Chem 58: 1630-1638, 2010.

31. Ohgidani M, Komizu Y, Goto K and Ueoka R: Residual powders from Shochu distillation remnants induce apoptosis in human hepatoma cells via the caspase-independent pathway. J Biosci Bioeng 114: 104-109, 2012.

32. Yu VW and Ho WS: Tetrandrine inhibits hepatocellular carcinoma cell growth through the caspase pathway and $\mathrm{G} 2 / \mathrm{M}$ phase. Oncol Rep 29: 2205-2210, 2013. 